\title{
Fernández de Oviedo \\ (Historia General y Natural de las Indias, Libro VI, cap. LI) y la etología clásica
}

Jesús Lens-Tuero

Universidad de Granada

El presente trabajo se propone llamar la atención sobre un notable texto de Fernández de Oviedo (Historia General y Natural de las Indias, Libro VI, cap. LI) que, en la medida que conocemos, no ha sido objeto de un comentario adecuado. En dicho texto se refiere el establecimiento de un pacto entre el hombre y el animal para asegurarse la supervivencia y mutuo beneficio, y la transformación de tal pacto en una relación de amistad y afecto. Tales relaciones pueden ser iluminadas desde la antropología clásica, en la que dichas categorías desempeñaron un papel no carente de relevancia.

El objeto de nuestro estudio es una parte de la famosa obra del cronista Fernández de Oviedo ${ }^{1}$ que, en nuestra opinión, aún no ha sido analizada en profundidad: "En esta nuestra isla Española andan muchos negros alzados que se han rebelado del servicio de los cristianos; y así para castigar los tales, como para asegurar los que quedan en las haciendas de los pobladores, andan algunas cuadrillas de españoles en busca de los levantados. Y entre los otros capitanes nuestros, anda un hidalgo, llamado Antonio de Sanct Miguel, natural de Ledesma, hombre de bien e valiente por su persona, al cual yo conozco; y éste puede haber pocos meses que, yendo con sus compañeros por las sierras de la villa de Sanct Joan de la Managua (que es en la mitad desta nuestra isla, a la parte desta costa del Sur), topó con un indio cimarrón o bravo que andaba en cueros, e con ciertas varas tostadas para pelear o matar algunos puercos cimarrones o salvajes, de los cuales hay innumerables en esta isla, de los que se han ido al monte de los que se trujeron de España. E traía este indio en su compañía una puerca e dos puercos mansos a él, e con aquella compañía hacía su vida e comía e dormía entre ellos, e había doce años, o más, que andaba alzado,e era ladino, e hablaba nuestra lengua castellana muy bien.

E como acaso este capitán e su gente dieron en este indio e su porcesca compañía, los cristianos mataron luego aquellos dos puercos e puer-

1 Manejamos la edición de J. Pérez de Tudela Bueso para la Biblioteca de Autores Españoles, vol. I, Madrid, 1959.

Tomo LIV, 1, 1997 
ca, en un instante, sin saber su propriedad o ejercicio de los dichos puercos e puerca, por poder reparar su hambre, que había días que no había comido carne. Cuya muerte de aquellos tres animales fué mucho pesar e dolor para aquel indio, e queriéndose informar el dicho capitán de su manera de vida e soledad, e qué hacía con aquellos puercos, o para qué los quería, respondió e dijo: "Estos puercos me daban a mí la vida e me mantenían e yo a ellos; eran mis amigos e mi buena compañía; el uno se llamaba tal nombre, e el otro se decía el tal, e la puerca se llamaba la tal (como él los tenía nombrados)". El un puerco decía que era muy gran ventor, e el otro era más recio, e de presa, e muy denodado; de forma que el uno hacía el oficio de sabueso, e el otro de lebrel, e la puerca era consorte e coadjutor de los dos cuando era el tiempo que convenía ayudarlos. E así como era de día, este indio salía de su rancho e decía a sus compañeros los puercos: "Ea, amigos, vamos a buscar de comer." E así lo hacían; e el ventor tomaba la delantera, e como daba en el viento, aguijaba a donde le parescía que debía ir, e seguíanle el otro puerco e la puerca, e tras ellos iba el indio. E como el ventor topaba el puerco bravo, asíase con él a la lucha, e comenzaban su batalla, mordiéndose; e como llegaba la compañía, dábanle los tres mucha priesa a bocados; e como llegaba el indio con sus varas, daba favor a sus compañeros, e con ellas le hería, al puerco cimarrón, e le mataban presto. El cual muerto, le abría el indio e daba las interioras a sus compañeros, e él encendía fuego con los palillos, como los indios lo usan, e asaba lo que le parescía, con que él comía; e lo restante del defunto animal, hecho pedazos, lo cargaba sobre los dos puercos e puerca con sus cuerdas de bejucos, e íbanse a su rancho do acostumbraban dormir esta compañía. E allí descargados, colgados los tasajos o partes del puerco muerto, lo comían poco a poco, en tanto que, de la manera que es dicho, mataban otros u otros puercos. $\mathrm{E}$ las noches, el dicho indio se acostaba entre aquella su bestial compañía, rascando horas al uno e horas al otro, regalándolos a la porcesca. E luego, otro día, si no tenían carne, o no hallabn hovos, o no era tiempo de tal fructa, el indio sabía hallar ciertas raíces con que daba de comer a aquella su compañía, e a él no le faltaba. Desta manera que es dicho, hacía su vida este indio en aquellos montes.

Después que el capitán Antonio de Sanct Miguel e sus compañeros hobieron oído e entendido la nueva e nunca antes oída semejante montería, pesóles mucho de haber muerto los puercos, e lleváronse el indio consigo a la cibdad de la Vega, donde al presente está. 
Y porque yo tengo por estilo en lo que no he visto dar mi descargo con testigos fidedignos, digo que desta nuestra cibdad de Sancto Domingo salió el reverendísimo señor obispo don Alonso de Fuenmayor, e fué la tierra adentro a visitar sus iglesias, e en la cibdad de la Vega estuvo algunos días, donde le contó lo que es dicho el mismo capitán Antonio de Sanct Miguel, e otros que con él se hallaron, e vido el dicho señor obispo el mismo indio. E después que tornó a esta cibdad este nuetro perlado, yo oí lo que es dicho a algunas personas de crédito, e para más me satisfacer, lo pregunté al mismo señor obispo, e me dijo que es muy gran verdad e muy público todo lo que es dicho, e que pasó de la misma manera que aquí lo he escripto. Parescióme tan grande novedad y tan varia lección, e tan apartado caso de cuanto está dicho, ni visto, ni escripto, que cuadra bien aquí aquel soneto, a lo menos los cuatro versos primeros, en que dice Francisco Petrarca:

\section{La gola, il sonno, et l'ociose piume hanno del mondo ogni vertú sbandita, ond'e dal corso suo quasi smarrita nostra natura vinta dal costume.}

Quiere decir: la gula, el sueño, e las ociosas plumas, o cama, han desterrado del mundo todas las virtudes, e han apartado de su curso, cuasi, a nuestra natura, vencida de la costumbre; porque el hombre es dedicado a la razón, en diferencia de los animales brutos que son carescientes della.

Ved, pues, si en estos animales se muestra esto claramente; pues seyendo los puercos para ser monteados, se convirtieron, con la costumbre, en ser monteros e hacer el oficio que no les competía, e el indio, siendo animal racional e humano hombre, se convertía en puerco, o hacía su vida bestial, de la forma que es dicho. Así que, esto procedía de la larga consuetud que aquel indio había ejercitado enseñando aquellas bestias en tal montería, pegándoseles una entrañable amistad al oficio, juntamente con la nescesidad de ser alimentados; e mezclándose con eso unos celos o envidia que constreñía esos puercos a matar los otros que topaban, porque su amo no pusiese amor en otros, ni les mostrase el oficio, como a ellos lo enseñó, para que pudiese desdeñarlos ni poner otros en su lugar. Y el indio, apartándose de la excelencia de la razón,y sin tener cuenta ni respeto ni temor a su Dios, huyendo de los hombres, se contentaba de vevir con bestias y ser bestial.

Cosa es la que he contado que a mí me dió mucha admiración oírla, y no la osara escrebir si no me certificara primero deste reverendísimo señor obispo, presidente de Sus Majestades en la Real Audiencia e Chan- 
cillería que reside en esta cibdad de Sancto Domingo, cuya auctoridad e persona es de tanto crédito, que, solo, bastaba para ser creído, no obstante la novedad de tal montería; cuanto más que otros muchos dicen lo mismo, por cosa muy pública e notoria en aquella cibdad de la Vega".

$$
* * *
$$

Para efectos del análisis conviene distinguir entre el relato propiamente dicho y la interpretación de su contenido que hace el cronista. La información, pues, le llega a Fernández de Oviedo del obispo don Alonso de Fuenmayor, quien había conocido personalmente al indio protagonista de la historia y al capitán Antonio de Sanct Miguel, cuyos hombres habían dado muerte a los puercos. El relato de los hechos, aunque cabe legítimamente suponer que Fernández de Oviedo lo ha conformado de acuerdo con su interpretación, puede ser leído de modo autónomo y ser objeto, a su vez, de una interpretación relativamente diferente. Tal lectura alternativa podría ir en la línea de subrayar la ruptura de límites entre la condición humana y la animal, que lleva, en este caso concreto, al establecimiento entre el hombre y los cerdos objeto del relato de una relación de amistad ("Esos puercos... eran mis amigos e mi buena compañía") y de afecto ("E las noches, el dicho indio se acostaba entre aquella su bestial compañía, rascando horas al uno e horas al otro, regalándolos a la porcesca"). Esa relación garantizaba a las partes implicadas la mutua supervivencia ("Esos puercos me daban a mí la vida e me mantenían e yo a ellos; E luego, otro día, si no tenían carne... el indio sabía hallar ciertas raíces con que daba de comer a aquella su compañía, e a él no le faltaba").

El establecimiento de un pacto entre el hombre y el animal para asegurarse la supervivencia y mutuo beneficio, y la transformación de tal pacto en una relación de amistad y afecto pueden ser iluminadas desde la antropología clásica, en la que dichas categorías desempeñaron un papel no carente de relevancia.

Existen, como es bien sabido, dos concepciones divergentes en el pensamiento antropológico grecorromano sobre la primigenia condición de la humanidad. Ambas coincidían en entender que la vida primitiva de los hombres estaba próxima a la de los animales, pero diferían radicalmente en la valoración de tal situación. Una corriente de opinión entendía que el hombre, en esta su primitiva su condición bestial, era víctima de los ataques 
de las fieras salvajes y de las inclemencias atmosféricas, inconvenientes de los que había logrado liberarse gracias al desarrollo de las técnicas, y en particular del fuego. ${ }^{2}$ En esta línea de subrayar la básica oposición entre hombre y animal cabe citar, a la inversa, un pasaje de Plutarco, ${ }^{3}$ que volveremos a mencionar al final de este trabajo, en el que se señala que la mayor parte de los animales rehuyen el contacto con el hombre, y que las golondrinas, aunque se introducen en las casas en busca de oscuridad y de la seguridad que necesitan, rehuyen y temen al hombre como a una bestia.

La otra corriente de opinión entendía que en esa primitiva condición bestial el hombre había conocido la auténtica felicidad, al vivir en conformidad con la naturaleza. Esta doctrina, aunque no sin ciertas ambigüedades, ${ }^{4}$ fue la de Platón. Como ha señalado muy bien U. Dierauer, ${ }^{5}$ en el mito del Político Platón presentaba el retrato de una etapa primitiva de la humanidad, situada bajo la advocación de Crono, en la que hombres y animales no se producían ningún daño, sino que incrementaban su sabiduría en mutua conversación. ${ }^{6}$ Platón, a este respecto, ha tenido un antecesor en Empédocles, en la obra del $\mathrm{cual}^{7}$ se nos aparece por vez primera la idea de que la fase originaria de la ausencia de culpa está caracterizada por la amistad y concordia de hombres y animales. Al igual que los animales no causaban daño a los hombres, tampoco éstos perjudicaban a los restantes seres vivos. En la cultura grecorromana el motivo de la paz con los animales no sólo desempeñó un papel en las representaciones de una pasada edad de Oro, sino también más adelante en las representaciones escatológicas.

Platón, pues, hacía una valoración totalmente negativa de la separación del hombre respecto del animal. ${ }^{8}$ La fabulación platónica del Político tiene en cualquier caso la interesante peculiaridad (anticipada en Empédocles) de que el mundo que retrata se caracteriza por una paz universal, que se extiende también al mundo animal, y que se manifiesta en que entre las bestias no existe guerra ni querella de ningún tipo. ${ }^{9}$

2 Dodds, E.R.: The ancient concept of progress and other essays on greek literature and belief, Oxford, 1973, págs. 1 y ss.

3 Moralia 984.

4 Que han sido bien puestas de manifiesto por Vidal-Naquet, P.: "Plato's myth of the statesman, the ambiguities of the Golden Age and of history”, JHS, 98, 1978, págs. 132 y ss.

5 Tier und Mensch im Denken der Antike, Amsterdam, 1977, pág. 31.

6 Pol. 271c-272d.

7 F 130 Diels-Kranz.

8 Como ha dicho acertadamente Vidal-Naquet en el trabajo que arriba citamos, pág. 138: "el paraiso de la Edad de Oro era de modo totalmente definido un paraiso animal".

9 Pol. 271d. 
Dentro de la misma línea de exaltación del primitivismo encontramos otras exposiciones en las que las relaciones entre hombre y animal comportan una íntima unión que no excluye, sin embargo, el consumo de carne animal. Son las concepciones que exaltan la vida del hombre conforme a la naturaleza, y tienen una visión crítica de las convenciones que han venido a sustituir o alterar ese natural modo de vida. Tal orientación ideológica era seguramente ya la de Demócrito, y la compartieron plenamente los cínicos. Como lo ha expresado muy bien Cole: 10 "los cínicos y Demócrito son aliados hasta un cierto punto, y esto es indudablemente lo que explica el uso por los cínicos de la doctrina de Demócrito...; los cínicos, al igual que Demócrito, están dispuestos a considerar todos los usos que prevalecen en el dominio político y social de la existencia humana como la obra de la convención más bien que de la naturaleza; al igual que Demócrito, los cínicos están ansiosos por establecer el hecho de que el hombre una vez vivió una vida que no podía distinguirse de la de los animales".

Si del ámbito de la reflexión filosófica pasamos al de la etnografía, encontramos un texto que posee un notable paralelismo con el de Fernández de Oviedo en los Relatos indios de Ctesias; se trata de un pasaje que nos ha sido conservado por Eliano, en su Sobre la naturaleza de los animales:" "Dice Ctesias en sus Relatos indios que los llamados Ordeñadores de perras crían muchos perros del tamaño de los hircanios, y que son excelentes criadores de perros. Y las razones que aduce el autor de Cnido son las siguientes: desde el solsticio de verano hasta mediados del invierno los invaden rebaños de bueyes, como un emjambre de abejas o un avispero puesto en movimiento; el número de estos bueyes es muy considerable; son salvajes y violentos, y amenazan terriblemente con sus cuernos. Como no pueden rechazarlos de otro modo, lanzan contra ellos los perros que crían con este objetivo, quienes los combaten y los matan con total facilidad. Luego separan los trozos de carne que les parecen adecuados para su consumo, y el resto lo reservan para los perros, compartiéndola con ellos de la mejor gana, como se ofrecen primicias a benefactores. Y en la época en que ya no vienen los bueyes, tienen a sus perros de compañeros de caza contra los otros animales. Y ordeñan la leche de las hembras, hecho del que derivan su nombre; pues la beben, como nosotros la leche de las ovejas y las cabras".

10 Democritus and the sources of greek anthropology, Cleveland, 1967, pág. 152.

11 XVI.31. Se trata del F. 46 de Ctesias en la edición de F. Jacoby. 
Con carácter general, el tema de la íntima comunidad de vida entre pueblos primitivos y el mundo animal parece haber interesado a la etnografía helenística. Agatárquides, en concreto, se ocupó de un pueblo africano primitivo que vivía en estrecho contacto con las focas. Perdida para nosotros la obra original de Agatárquides, hemos de recuperar su exposición a través de los resúmenes de Diodoro ${ }^{12}$ y Focio. ${ }^{13}$ Según Diodoro se trata del pueblo de los Ictiófagos (es decir, Comedores de peces) apáticos, en compañía de los cuales viven las focas en cuestión, "que capturan peces por su cuenta de manera semejante a como lo hacen los hombres. De modo similar en lo que se refiere a los lechos, los nacimientos y la protección de los recién nacidos tienen relaciones recíprocas caracterizadas por la mayor lealtad; porque la convivencia de seres de estirpes diferentes se verifica sin injusticia, con paz y el máximo respeto. Este modo de vida, aunque resulte sorprendente, es observado desde los tiempos antiguos por estas razas, adaptado o bien a una habituación debida al tiempo, o bien a una necesidad impuesta por las circunstancias".

En el resumen de Focio el pueblo que convivía con las focas era uno próximo al de los Ictiófagos, y la relación era expuesta de modo muy resumido:"viven entre ellos de un modo tal como rara vez se vería a hombres vivir con hombres".

Incluso en una forma tan resumida, el paralelo entre el texto de Agatárquides y el de Fernández de Oviedo resulta notable, tanto en los detalles de la convivencia como en lo que concierne a la interpretación. En el primer plano destaca la coincidencia en la intimidad de la relación, que supera la cooperación en la alimentación para extenderse a compartir los lechos. En el segundo plano destaca sobre todo la común insistencia en la habituación como categoría explicativa de las conductas practicadas por todas las partes implicadas (Agatárquides en Diodoro: "Este modo de vida... adaptado o bien a una habituación debida al tiempo, o bien a una necesidad impuesta por las circunstancias; Fernández de Oviedo: Esto procedía de la larga consuetud que aquel indio había ejercitado enseñando aquellas bestias en tal montería").

También merece la pena hacer notar el modo en que sus respectivos autores subrayan el carácter paradoxográfico de sus narraciones; así Agatárquides hacía notar (en la versión de Diodoro) que "este modo de

12 III. 18.7.

13450 b 20-451 a 4 . 
vida, aunque resulte sorprendente, es observado desde los tiempos antiguos por estas razas y Fernández de Oviedo indicaba que cosa es la que he contado que a mí me dió mucha admiración oírla, y no la osara escrebir si no me certificara..."

Algún autor griego llegaba más lejos, y sostenía que algunos indios se unían con bestias, y tenían una progenitura híbrida y semibestial. Tal información, procedente del historiador griego Duris, la recogía Plinio en su Historia Natural..$^{14}$ Duris era un historiador griego del siglo IV a.C. que parece haber tenido un interés particular por las manifestaciones anormales de la sexualidad; así, había sostenido, en contra de la tradición acreditada por la Odisea, que Penélope se había unido sexualmente a todos los pretendientes, unión de la que había nacido Pan (=todo, en griego).${ }^{15}$

Pero el objetivo fundamental de este trabajo no estriba en hacer depender este texto de la tradición grecorromana, sino en mostrar en qué medida ésta puede iluminarlo. En las representaciones clásicas del primitivismo se ponen de relieve algunos aspectos que nos hacen reparar, y poner la debida atención, en otros similares del relato de Fernández de Oviedo. La vida del indio protagonista de éste transcurre en la montaña: "Antonio de Sanct Miguel... yendo con sus compañeros por las sierras de Sanct Joan de la Managua... topó con un indio cimarrón o bravo;... Desta manera que es dicho, hacía su vida este indio en aquellos montes". Como hace notar recientemente R.G.A. Buxton ${ }^{16}$ en las montañas imaginarias, las relaciones sociales y el comportamiento social normal pueden ser invertidos. Estas formas posibles de inversión se localizan preferentemente en las montañas, en un cierto sentido, porque la vida en ellas está presidida, al menos en un grado importante, por la soledad: "E queriéndose informar el dicho capitán de su manera de vida e soledad". Así, por ejemplo, en un papiro de tendencia cínica leemos: ${ }^{17}$ "Nos complacemos en nuestra soledad habitando entre los árboles... No necesitamos tener una ciudad, nido de hombres insidiosos. Dios construyó casa para nosotros:montes y bosque".

14 VII. 30: Duris Indorum quosdam cum feris coire mixtosque et semiferos esse partus.

15 F 21 Jacoby: "Y Duris en su Historia de Agatocles dice que Penélope fue lasciva, y que habiendo mantenido relaciones sexuales con todos los pretendientes, dió a luz a Pan de pata de cabra, que es considerado uno de los dioses".

16 "Montagnes mythiques, montagnes tragiques", Ktema, 15, 1990, pág. 168

17 Puede ser leído, tanto en griego como en español, en Oliver Segura, J.P.: "Diálogo del rey Alejandro con el brahmán Dándamis”, en F. Gascó y J. Alvar (ed.): Heterodoxos, reformadores y marginados en la Antigüedad clásica, Sevilla, 1991, págs. 107 y ss. 
Retornando ahora al texto de Fernández de Oviedo, constatamos que su interpretación no va totalmente en el sentido de exaltación de la vida primitiva. La línea maestra de su exégesis no es la de exaltación de un primitivismo feliz (vida del hombre conforme con la naturaleza) frente a las complicaciones y dificultades que han aportado los elementos superfluos (la convención), sino que el concepto básico desde el que interpreta este relato es el de adaptación: "el indio, siendo animal racional e humano hombre, se convertía en puerco, o hacía su vida bestial. Así que, esto procedía de la larga consuetud que aquel indio había ejercitado enseñando aquellas bestias en tal montería, pegándoseles una entrañable amistad al oficio, juntamente con la necesidad de ser alimentados".

Esta interpretación es posible, y enseguida vamos a ver que tiene antecedentes también en el pensamiento antropológico grecorromano. Lo interesante es que, como apoyo de su interpretación, Fernández de Oviedo aduce un texto de Petrarca, muy conocido, ${ }^{18}$ recogido en la pág. 187 (y $3 .{ }^{\mathrm{a}}$ de este artículo), que se adecua mucho mejor a la primera exégesis posible, la que el cronista no ha seguido.

El costume del italiano es entendido por Fernández de Oviedo como habituación. Entender la palabra costume como costumbre es ciertamente posible, pero el contraste que el gran poeta establecía era mucho más probablemente el de la naturaleza vencida por la convención. Esta lectura, que es la más adecuada de estos versos tomados aisladamente, gana todavía mucha más credibilidad cuando leemos el poema completo, dominado por el contraste entre la simplicidad y lo superfluo.

El poema de Petrarca, y en particular los cuatro versos citados por Fernández de Oviedo, se adecuan particularmente bien a una lectura de la historia en clave primitivista de exaltación de la vida conforme a la naturaleza, y es posible que relato y poema hayan llegados juntos a Fernández de Oviedo. Este, cuya orientación ideológica general no es la de exaltación del primitivismo, ha interpretado el relato en clave de habituación: la consuetud que aparece expresamente en el texto que hemos citado. Esta "larga consuetud que aquel indio había ejercitado" poseía tres componentes: en primer lugar "enseñando aquellas bestias en tal montería, pegándoseles una entrañable amistad al oficio"; en segundo lugar, "juntamente con la necesidad de ser alimentados"; en tercer lugar, "e mezclándose con eso unos celos o envidia que constreñía esos puercos a matar los otros que topaban,

18 Cancionero I.7. 
porque su amo no puesiese amor en otros, ni les mostrase el oficio, como a ellos lo enseñó, para que pudiese desdeñarlos ni poner otros en su lugar".

Entre el indio y sus cerdos domésticos se había establecido, pues, una larga consuetud basada, en primer lugar, en compartir una misma actividad; en segundo, en la común búsqueda de alimentación y, en tercer lugar, en el establecimiento de una profunda relación afectiva, que llevaba a los cerdos a buscar la eliminación de cualquier posible rival.

También a este respecto podemos aducir un sugestivo paralelo de la Antigüedad grecorromana. Jenofonte, como nos recuerda Cole, ${ }^{19}$ practicó un análisis utilitarista de la moralidad social, siendo las Memorabilia un locus classicus en la Antigüedad para este punto de vista, y su teoría ética mezcla lo utile y lo dulce. Proximidad y hábitos comunes crean afecto, y el proceso es observable en la conducta tanto de hombres como de animales. De los textos aducidos por Cole el más interesante es el segundo de Ciropedia: "Y le parecía que el hecho de comer juntos ayudaba a que tuvieran menos deseos de abandonarse los unos a los otros, porque veía que los animales que son alimentadoa juntos tienen una terrible pena si se los separa".

De otra parte, como nos recuerda el mismo Cole, ${ }^{20}$ en el libro VI de sus Historias Polibio incluye, como una especie de prefacio a su teoría cíclica de la transformación de las constituciones, un relato del desarrollo de la sociedad desde sus comienzos tipo horda hasta la creación de la monarquía (término con el que Polibio designa, en realidad, al primer gobierno basado en el consenso popular). Las conceptualizaciones a este respecto utilizadas por Polibio son las de syntrophía y synétheia, comunidad de comida y comunidad de actividad: "Cuando, con el paso del tiempo, surge dentro de estas agregaciones una alimentación común (syntrophía) y un modo de vida común (synétheia), éste es el comienzo natural de la monarquía, y entonces es cuando por vez primera surge entre los hombres una noción de lo correcto y de lo justo, e igualmente de sus opuestos".

El paso de la asociación, consecuencia de la syntrophía y la synétheia, al desarrollo de una noción de lo correcto y de lo justo lo dibuja Polibio como un proceso sicológico, en virtud del cual la comunidad manifiesta su rechazo respecto, en particular, de las conductas ingratas.

19 Democritus and the sources of greek anthropology, pág. 132.

20 Ibídem, pág. 80.

21 VI. 5. 10. 
Los efectos de la syntrophía y la synétheia serían especialmente pronunciados en donde la asociación entre los individuos es más estrecha, en la familia; tanto en el rebaño humano como en el animal existen ciertas tendencias hacia la conducta recíproca que, acentuadas y expandidas, son capaces de producir sociedad.

Resulta interesante anotar que cabe establecer un paralelo muy estrecho entre las tres categorías polibianas y las de Fernández de Oviedo. La "entrañable amistad al oficio" que se le pegaba a las bestias bajo la enseñanza del indio es un buen correlato de la synétheia polibiana; la categoría de la syntrophía polibiana, la comunidad de alimentación, está muy subrayada, como hemos visto reiteradamente, en el relato del cronista de Indias. Insistimos ahora en el paralelismo entre ambos textos en la dimensión sicológica de la cohesión que a los grupos respectivamente tomados en consideración les procura su relación con los otros miembros del mismo.

De modo, pues, que en los dos textos que consideramos se genera una asociación en base a la comunidad de actividad, la comunidad de comida y la comunidad de afectos.

En el escrito en el que Plutarco se interesa por la inteligencia de los animales, ${ }^{22}$ los conceptos básicos utilizados para caracterizar las relaciones entre el mundo humano y el animal son también los de alimentación y convivencia. Plutarco anota ${ }^{23}$ que de los animales de tierra algunos evitan absolutamente al hombre, mientras que otros, los más domesticados, dan buen trato a quienes los alimentan, y el perro, el caballo y el elefante tratan especialmente bien a los hombres con quienes tienen familiaridad. Son las dos categorías básicas del relato de Fernández de Oviedo, la alimentación, por un lado, y la familiaridad producida por el trato, de otro.

Hay, sin embargo, una importante diferencia entre los textos que hemos aducido de la tradición etnográfica grecorromana y el citado por Fernández de Oviedo, y es que en el caso de los dos primeros los hombres y una determinada especie animal (en un caso perros y en otro focas) establecen una convivencia para capturar animales de especies diferentes (en un caso bóvidos y en otro peces) de los que se alimentan. El texto citado por Fernández de Oviedo posee la peculiaridad excepcional de que los animales con los que el indio se había asociado pertenecen a la misma especie que los atacados: son cerdos unos y otros. Esta peculiaridad es lo que

22 Moralia 959 y ss.

23 Ibídem, 984c. 
permite al cronista de Indias desarrollar un aspecto ausente de los relatos grecorromanos. En efecto, la actuación mortífera de los cerdos compañeros del indio no está provocada únicamente por la necesidad de procurarse el alimento, sino también por el temor a que otros ejemplares de la especie puedan suplantarles en el afecto del hombre. Este punto parece ser fundamental, y no accesorio, en el relato citado por Fernández de Oviedo, y quizás se encuentre aquí la razón de que los animales seleccionados hayan sido, de modo un poco sorprendente, cerdos y no perros, que son los animales más adecuados para el establecimiento de relaciones estrechas entre hombres y animales con vistas a procurarse la alimentación. 